\title{
The fundamental nature of space and time*
}

\author{
Gerard 't Hooft \\ Institute for Theoretical Physics \\ Utrecht University \\ and \\ Spinoza Institute \\ Postbox 80.195 \\ 3508 TD Utrecht, the Netherlands \\ e-mail: g.thooft@uu.nl \\ internet: http://www.phys.uu.nl/ thooft/
}

\begin{abstract}
Both the Standard Model of elementary particle physics and the classical theory of General Relativity ore observed to obey the fundamental requirements of causality and locality. Most of the recent attempts at reconciling these two theories into one, assume the standard axioms of quantum mechanics as well as those of general coordinate invariance without further question, whereas the demands of locality and even causality are often compromised upon. Background independence is usually sought for. There are reasons to suspect that this will not yield a comprehensive formalism. In particular, black holes lead to conceptual difficulties, in view of the holographic nature of the horizon. The author advocates more imaginative scenarios. The most extreme possibility is an "underlying theory", with neither general relativity nor quantum mechanics in its basic equations, both having an "emergent" nature.
\end{abstract}

*Submitted for publication in Quantum Gravity, Cambridge University Press, Daniele Oriti, Ed. 


\section{Quantum Gravity as a non-renormalizable gauge theory}

Quantum gravity is usually thought of as a theory, under construction, where the postulates of quantum mechanics are to be reconciled with those of general relativity, without allowing for any compromise in either of the two. As will be argued in this contribution, this 'conservative' approach may lead to unwelcome compromises concerning locality and even causality, while more delicate and logically more appealing schemes can be imagined.

The conservative procedure, however, must first be examined closely. The first attempt (both historically and logically the first one) is to formulate the theory of "quantum gravity" perturbatively[1], as has been familiar practice in the quantum field theories for the fundamental particles, vis. the Standard Model. In perturbative quantum gravity, one takes the Einstein-Hilbert action,

$$
S=\int \mathrm{d}^{4} x \sqrt{-g}\left(\frac{R(x)}{\varepsilon}+\mathcal{L}^{\text {matter }}(x)\right), \quad \varepsilon=16 \pi G,
$$

considers the metric to be close to some background value: $g_{\mu \nu}=g_{\mu \nu}^{\mathrm{Bg}}+$ $\sqrt{\varepsilon} h_{\mu \nu}$, and expands everything in powers of $\varepsilon$, or equivalently, Newton's constant $G$.

Invariance under local coordinate transformations then manifests itself as a local gauge symmetry: $h_{\mu \nu} \rightarrow h_{\mu \nu}+D_{\mu} u_{\nu}+D_{\nu} u_{\mu}$, where $D_{\mu}$ is the usual covariant derivative, and $u_{\mu}(x)$ generates an infinitesimal coordinate transformation. Here one can use the elaborate machinery that has been developed for the Yang-Mills theories of the fundamental particles. After imposing an appropriate gauge choice, all desired amplitudes can be characterized in terms of Feynman diagrams. Usually, these contain contributions of 'ghosts', which are gauge dependent degrees of freedom that propagate according to well-established rules. At first sight, therefore, quantum gravity does not look altogether different from a Yang-Mills theory. It appears that at least the difficulties of reconciling quantum mechanics with general coordinate invariance have been dealt with. We understand exactly how the problem of time, of Cauchy surfaces, and of picking physical degrees of freedom, are to be handled in such a formalism. Indeed, unitarity is guaranteed in this formalism, and, in contrast to 'more advanced' schemes for quantizing gravity, the perturbative approach can deal adequately with problems such as: what is the complete Hilbert space of physical states?, how can the fluctuations of the light cone be squared with causality?, etc., simply because at all finite orders in per- 
turbation expansion, such serious problems do not show up. Indeed, this is somewhat surprising, because the theory produces useful amplitudes at all orders of the perturbation parameter $\varepsilon$.

Yet there is a huge difference with the Standard Model. This 'quantum gauge theory of gravity' is not renormalizable. We must distinguish the technical difficulty from the physical one. Technically, the 'disaster' of having a non-renormalizable theory is not so worrisome. In computing the $\mathcal{O}\left(\varepsilon^{n}\right)$ corrections to some amplitude, one has to establish $\mathcal{O}\left(\varepsilon^{n}\right)$ correction terms to the Lagrangian, which are typically of the form $\sqrt{-g} R^{n+1}$, where $n+1$ factors linear in the Riemann curvature $R_{\beta \mu \nu}^{\alpha}$ may have been contracted in various possible ways. These terms are necessary to cancel out infinite counter terms of this form, where finite parts are left over. At high orders $n$, there exist many different expressions of the form $R^{n+1}$, which will all be needed. This is often presented as a problem, but, in principle, it is not. It simply means that our theory has an infinite sequence of free parameters, not unlike many other theories in science, and it nevertheless gives accurate and useful predictions op to arbitrarily high powers of $G E^{2}$, where $E$ is the energy scale considered. We emphasize that this is actually much better than many of the alternative approaches to quantum gravity such as loop quantum gravity, and even string theory presents us with formidable problems when 3-loop amplitudes are asked for. Also, claims[2] that quantum gravity effects might cause 'decoherence' at some finite order of $G E^{2}$ are invalid according to this theory.

Physically, however, the perturbative approach fails. The difficulty is not the fact that the finite parts of the counter terms can be freely chosen. The difficulty is a combination of two features: $(i)$ perturbation expansion does not converge, and ( $i i)$ the expansion parameter becomes large if center-of-mass energies reach beyond the Planck value. The latter situation is very reminiscent of the old weak interaction theory where a quartic interaction was assumed among the fermionic fields. This Fermi theory was also "non-renormalizable".

In the Fermi theory, this problem was solved: the theory was replaced by a Yang-Mills theory with Brout-Englert-Higgs mechanism. This was not just 'a way to deal with the infinities', it was actually an answer to an absolutely crucial question[3]: what happens at small distance scales?. At small distance scales, we do not have quartic interactions among fermionic fields, we have a local gauge theory instead. This is actually also the superior way to phrase the problem of quantum gravity: What happens at, or beyond, the Planck scale? Superstring theory[4] is amazingly evasive if it comes to considering this question. It is here that Loop Quantum Gravity[5] appears to be the most direct approach. It is an attempt to 
characterize the local degrees of freedom, but is it good enough?

\section{A prototype: gravitating point particles in $2+1$ dimensions}

An instructive exercise is to consider gravity in less than four space-time dimensions. Indeed, removing two dimensions allows one to formulate renormalizable models with local diffeomorphism invariance. Models of this sort, having one space- and one time dimension, are at the core of (super)string theory, where they describe the string world sheet. In such a model, however, there is no large distance limit with conventional 'gravity', so it does not give us hints how to cure non-renormalizable longdistance features by modifying its small distance characteristics. There is also another reason why these 2-dimensional models are uncharacteristic for conventional gravity: formally, pure gravity in $d=2$ dimensions has $\frac{1}{2} d(d-3)=-1$ physical degrees of freedom, which means that an additional scalar field is needed to turn the theory into a topological theory. Conformal symmetry removes one further degree of freedom, so that, if string theory starts with $D$ target space variables, or 'fields', $X^{\mu}(\sigma, \tau)$, where $\mu=1, \cdots, D$, only $D-2$ physical fields remain.

For the present discussion it is therefore more useful to remove just one dimension. Start with gravitationally interacting point particles in 2 space dimensions and one time. The classical theory is exactly solvable, and this makes it very interesting. Gravity itself, having zero physical degrees of freedom, is just topological; there are no gravitons, so the physical degrees of freedom are fust the gravitating point particles. In the large distance limit, where quantum mechanical effects may be ignored, the particles are just point defects surrounded by locally flat space-time. The dynamics of these point defects has been studied[6], and the evolution laws during finite time intervals are completely understood. During very long time intervals, however, chaotic behavior sets in, and also, establishing a complete list of all distinguishable physical states turns out to be a problem. One might have thought that quantizing a classically solvable model is straightforward, but it is far from that, exactly because of the completeness problem. $2+1$ gravity without point particles could be quantized[7], but that is a topological theory, with no local degrees of freedom; all that is being quantized are the boundary conditions, whatever that means.

One would like to represent the (non-rotating) point particles by some scalar field theory, but the problems one then encounters appear to be 
formidable. Quite generally, in 2+1 dimensions, the curvature of 2-space is described by defect angles when following closed curves (holonomies). The total defect angle accumulated by a given closed curve always equals the total matter-energy enclosed by the curve. In the classical model, all of this is crystal clear. But what happens when one attempts to 'quantize' it? The matter Hamiltonian density does not commute with any of the particle degrees of freedom, since the latter evolve as a function of time. Thus, anything that moves, is moving in a space-time whose curvature is non-commuting. This is an impediment against a proper formulation of the Hilbert space in question in the conventional manner. Only eigenstates of the Hamiltonian and the Hamiltonian density can live in a 2-space with precisely defined 2-metric. Consequently, if we wish to describe physical states in a 2-space with precisely defined metric, these states must be smeared over a period of time that is large compared to the Planck time. We repeat: in a perturbative setting this situation can be handled because the deviations from flat space-time are small, but in a non-perturbative case, we have to worry about the limits of the curvature. The deficit angles cannot exceed the value $2 \pi$, and this implies that the Hamilton density must be bounded.

There is, however, an unconventional quantization procedure that seems to be quite appropriate here. We just noted that the Hamiltonian of this theory is unmistakably an angle, and this implies that time, its conjugate variable, must become discrete as soon as we quantize. Having finite time jumps clearly indicates in what direction we should search for a satisfactory quantum model: Schrödinger's equation will be a finite difference equation in the time direction. Take that as a modified picture for the small-distance structure of the theory!

How much more complicated will the small-distance structure be in our $3+1$ dimensional world? Here, the Hamiltonian is not limited to be an angle, so, time will surely be continuous. However, if we restrict ourselves to a region where one or more spatial dimensions are taken to be confined, or compactified, taking values smaller than some scale $L$ in Planck units, then it is easy to see that we are back in the $2+1$ dimensional case, the Hamiltonian is again an angle, and time will be quantized. However, the $2+1$ dimensional Newton's constant will scale like $1 / L$, and the time quantum will therefore be of order $1 / L$ in Planck units. This suggests the following. In finite slabs of 3-space, time is quantized, the states are 'updated' in discretized time steps. If we stitch two equal sized slabs together, producing a slab twice as thick, then updating happens twice as fast, which we interpret as if updating happens alternatingly in one slab and in the other. The total time quantum has decreased by a factor two, 
but within each slab, time is still quantized in the original units. The picture we get this way is amazingly reminiscent of a computer model, where the computer splits 3-space into slabs of one Planck length thick, and during one Planck time interval every slab is being updated; a stack of $N$ slabs thus requires $N$ updates per Planck unit of time.

\section{Black holes, causality and locality}

The $2+1$ dimensonal theory does not allow for the presence of black holes (assuming a vanishing cosmological constant, as we will do throughout). The black hole problem, there, is simply replaced by the restriction that the energy must stay less than the Planck value. In our slab-stack theory (for want of a better name), we see that the energy in every slab is restricted to be less than the Planck value, so any system where one of the linear dimensions is less than $L$, should have energy less than $L$ in Planck units, and this amounts to having a limit for the total energy that is such that a black hole corresponds to the maximally allowed energy in a given region.

Clearly, black holes will be an essential element in any quantum gravity theory. We must understand how to deal with the requirement that the situation obtained after some gravitational collapse can be either described as some superdense blob of mass and energy, or as a geometric region of space-time itself where ingoing observers should be allowed to apply conventional laws of physics to describe what they see.

One can go a long way to deduce the consequences of this requirement. Particles going into a black hole will interact with all particles going out. Of all these interactions, the gravitational one happens to play a most crucial role. Only by taking this interaction into account[8], can one understand how black holes can play the role of resonances in a unitary scattering process where ingoing particles form black holes and outgoing particles are the ones generated by the Hawking process.

Yet how to understand the statistical origin of the Hawking-Bekenstein entropy of a black hole in this general framework is still somewhat mysterious. Even if black hole entropy can be understood in superstring theories for black holes that are near extremality, a deep mystery concerning locality and causality for the evolution laws of Nature's degrees of freedom remains. Holography tells us that the quantum states can be enumerated by aligning them along a planar surface. The slab-stack theory tells us how often these degrees of freedom are updated per unit of time. How do we combine all this in one comprehensive theory, and how can we reconcile 
this very exotic numerology with causality and locality? May we simply abandon attempts to rescue any form of locality in the $3+1$ dimensional bulk theory, replacing it by locality on the dual system, as is done in the AdS/CFT approach[9] of $M$-theory?

\section{The only logical way out: deterministic quantum mechanics}

It is this author's opinion that the abstract and indirect formalisms provided by $M$-theory approaches are unsatisfactory. In particle physics, the Standard Model was superior to the old Fermi theory just because it provided detailed understanding of the small-distance structure. The small distance structure of the $3+1$ dimensional theory is what we wish to understand. The holographic picture suggests discreteness in space, and the slab-stack theory suggests discreteness in time. Together, they suggest that the ultimate laws of Nature are akin to a cellular automaton[10].

However, our numerology admits far fewer physical states than one (discrete) degree of freedom per unit of bulk volume element. We could start with one degree of freedom for every unit volume element, but then a huge local symmetry constraint would be needed to reduce this to physical degrees of freedom which can be limited to the surface. This situation reminds us of topological gauge theories. How will we ever be able to impose such strong symmetry principles on a world that is as non-trivial as our real universe? How can we accommodate for the fact that the vast majority of the 'bulk states' of a theory should be made unphysical, like local gauge degrees of freedom?

Let us return to the $2+1$ dimensional case. Suppose that we would try to set up a functional integral expression for the quantum amplitudes. What are the degrees of freedom inside the functional integrand? One would expect these to be the defects in a space-time that is flat everywhere except in the defects. A defect is then characterized by the element of the Poincaré group associated with a closed loop around the defect, the holonomy of the defect. Now this would force the defect to follow a straight path in space-time. It is not, as in the usual functional integral, an arbitrary function of time, but, even inside the functional integral, it is limited to straight paths only. Now this brings us back from the quantum theory to a deterministic theory; only deterministic paths appear to be allowed. It is here that this author thinks we should search for the clue towards the solution to the aforementioned problems. 
The topic that we dubbed "deterministic quantum mechanics" [11] is not a modification of standard quantum mechanics, but must be regarded as a special case. A short summary, to be explained in more detail below, is that our conventional Hilbert space is part of a bigger Hilbert space; conventional Hilbert Space is obtained from the larger space by the action of some projection operator. The states that are projected out are the ones we call "unphysical", to be compared with the ghosts in local gauge theories, or the bulk states as opposed to the surface states in a holographic formulation. In the bigger Hilbert space, a basis can be found such that basis elements evolve into basis elements, without any quantum mechanical superposition ever taking place.

One of the simplest examples where one can demonstrate this idea is the harmonic oscillator, consisting of states $|n\rangle, n=0,1, \cdots$, and

$$
H|n\rangle=\left(n+\frac{1}{2}\right)|n\rangle .
$$

If we add to this Hilbert space the states $|n\rangle$ with $n=-1,-2, \cdots$, on which the Hamiltonian acts just as in Eq. (4.1), then our ontological basis consists of the states

$$
|\varphi\rangle=\frac{1}{\sqrt{2 N+1}} \sum_{n=-N}^{N} e^{-i n \varphi}|n\rangle,
$$

which evolve as

$$
|\varphi\rangle \underset{t=T}{\longrightarrow}|\varphi+T\rangle
$$

provided that $(2 N+1) T / 2 \pi$ is an integer. In the limit $N \rightarrow \infty$, time $T$ can be taken to be continuous. In this sense, a quantum harmonic oscillator can be turned into a deterministic system, since, in Eq. (4.3) the wave function does not spread out, and there is no interference. A functional integral expression for this evolution would only require a single path, much as in the case of the $2+1$ dimensional defects as described above. Since $\varphi$ is periodic, the evolution (4.3) describes a periodic motion with period $T=2 \pi$. Indeed, every periodic deterministic system can be mapped onto the quantum harmonic oscillator provided that we project out the elements of Hilbert space that have negative energy.

In general, any deterministic system evolves according to a law of the form

$$
\frac{\mathrm{d}}{\mathrm{d} t} q^{a}(t)=f^{a}(\vec{q}(t))
$$


(provided that time is taken to be continuous), and in its larger Hilbert space, the Hamiltonian is

$$
H=\sum_{a} f^{a} p_{a}, \quad p_{a} \stackrel{\text { def }}{=}-i \frac{\partial}{\partial q^{a}}
$$

where, in spite of the classical nature of the physical system, we defined $p_{a}$ as quantum operators. In this large Hilbert space, one always sees as many negative as positive eigenstates of $H$, so it will always be necessary to project out states. A very fundamental difficulty is now how to construct a theory where not only the negative energy states can be projected out, but where also the entire system can be seen as a conglomeration of weakly interacting parts (one may either think of neighboring sectors of the universe, or of weakly interacting particles), such that also in these parts only the positive energy sectors matter. The entire Hamiltonian is conserved, but the Hamilton densities, or the partial Hamiltonians, are not, and interacting parts could easily mix positive energy states with negative energy states. Deterministic quantum mechanics will only be useful if systems can be found where all states in which parts occur with negative energy, can also be projected out. The subset of Hilbert space where all bits and pieces only carry positive energy is only a very tiny section of the entire Hilbert space, and we will have to demonstrate that a theory exists where this sector evolves all by itself, even in the presence of non-trivial interactions.

What kind of mechanism can it be that greatly reduces the set of physical states? It is here that our self-imposed restriction to have strictly deterministic Hamilton equations may now bear fruit. In a deterministic system, we may have information loss. In a quantum world, reducing the dimensionality of Hilbert space would lead to loss of unitarity, but in a deterministic world there is no logical impediment that forbids the possibility that two different initial states may both evolve into the same final state.

This gives us a new view on what was once introduced as the 'holographic principle'. According to this principle, the number of independent physical variables in a given volume actually scales with the surface area rather than the volume. This may mean that, in every volume element, information concerning the interior dissipates away due to information loss, while only the information located on the surface survives, possibly because it stays in contact with the outside world.

Information loss forces us to assemble physical states in 'equivalence classes'. Two states are in the same equivalence classes if, in due time, they eventually evolve into the same final state. Equivalence classes may 
play the role of gauge equivalence classes, and thus we might arrive at a plausible scenario in which the degrees of freedom inside the bulk of some region are reduced to being gauge degrees of freedom, while the physical degrees of freedom are limited to reside on the surface.

Note that, if such a theory can be constructed, the 'primordial' laws of physics may be completely local and causal, but the physical states that figure in the evolution equation (4.4) appear to have a non-local definition. This may be the reason why more direct attempts to interpret quantum mechanical phenomena in terms of realistic theories tend to lead to a mysterious, invisible kind of non-locality, as laid down in the well-known Einstein-Podolsky-Rosen paradox.

\section{Information loss and projection}

How could information loss act as a mechanism to select out only those states where all energies are non negative? How exactly this works is not understood; however, we do have an instructive but admittedly vague argument, and it is the following. Consider several regions or systems in our universe that are only weakly interacting with one another. With the interaction switched off, they all obey deterministic evolution equations, and therefore, their Hamiltonians, which are of the form (4.5), have positive energy eigenvalues $E(i)_{a}$ and negative energy eigenvalues $-E(i)_{a}$, where (i) enumerates the systems and $a$ the eigenvalues. The combination of these systems will again have positive eigenvalues $E^{\text {tot }}=\sum_{i} E(i)_{a(i)}$ and negative energy eigenvalues $-E^{\text {tot }}$, but interactions must be arranged in such a way that all states where some energies are positive and some are negative are suppressed. The reason why we do allow all energies to be negative is that this might describe the physical situation equally well; we then happen to be dealing with the bra states $\langle\psi|$ rather than the kets $|\psi\rangle$.

Let us examine more closely the (weak) interaction between two of such systems. Consider a time interval $\delta t_{1}$ for system (1) and $\delta t_{2}$ for system (2). As argued earlier, both systems must be spread over many Planck time units. According to the uncertainty relation, let us assume that

$$
\begin{aligned}
\frac{1}{2}(E(1)+E(2)) & \approx \frac{1}{2\left(\delta t_{1}+\delta t_{2}\right)} ; \\
\frac{1}{2}|E(1)-E(2)| & \approx \frac{1}{2\left|\delta t_{1}-\delta t_{2}\right|} .
\end{aligned}
$$


Now, according to Eq. (4.3), unvertainty in time directly reflects uncertainty in the position $\varphi$ of the system in its periodic orbit. Demanding $E(1) E(2)>0$ corresponds to

$$
\begin{array}{rlrl}
(E(1)+E(2))^{2} & >(E(1)-E(2))^{2}, & \text { so that } \\
\left(\delta t_{1}+\delta t_{2}\right)^{2} & <\left(\delta t_{1}-\delta t_{2}\right)^{2}, & & \text { or } \\
\left(\delta \varphi_{1}+\delta \varphi_{2}\right)^{2} & <\left(\delta \varphi_{1}-\delta \varphi_{2}\right)^{2} . & &
\end{array}
$$

The details concerning the relative position $\delta \varphi_{1}-\delta \varphi_{2}$ wash away after a sufficiently large average time interval $\frac{1}{2}\left(\delta t_{1}+\delta t_{2}\right)$. We read off:

$$
\delta t_{1} \delta t_{2}<0
$$

Thus, the states that we expect to dissipate away due to information loss, are all those states where a positive time lapse $\delta t_{1}$ for one state is associated with a positive time lapse $\delta t_{2}$ for the other state. This may mean that the two states each carry an internal clock. The relative clock speed is controlled by the gravitational potential between the two systems. This potential apparently fluctuates. These fluctuations wash out all information concerning the relative configurations, but the relative clock speeds are always positive.

\section{The vacuum state and the Cosmological Constant}

We see that if we have a set of different systems which mutually interact only weakly, such as a set of free particles, or a set of disconnected pieces of the universe, either all energies must be selected to be positive, or they all are negative. This means that there is one very special state where all energies are zero: the vacuum state. Identifying the vacuum state is particularly difficult in our theory, but it seems that the vacuum also poses problems in other approaches. In loop quantum gravity, it is notoriously difficult to say exactly what the vacuum state is in terms of the fundamental loop states that were introduced there. In superstring theory, there are many candidates for the vacuum, all being distinctly characterized by the boundary conditions and the fluxes present in the compactified part of space-time. String theory ends up leaving an entire 'landscape' of vacuum states with no further indication as to which of these to pick. It is of crucial importance in any viable theory of Planck length physics to identify and describe in detail the vacuum state. It appears to be associated with very special fluctuations and correlations of 
the virtual particles and fields that one wishes to use to describe physical excited states, and the particles in it.

There exists an important piece of information telling us that the vacuum is not just the state with lowest energy. There must exist an additional criterion to identify the vacuum: it is flat - or nearly so. In perturbative gravity, this cannot be understood. The cosmological constant should receive a large finite renormalization counter term from all virtual interactions in the very high energy domain. A superior theory in which the cosmological constant vanishes naturally (or is limited to extremely tiny values) has not yet been found or agreed upon[12]. This should be a natural property of the vacuum state. To see most clearly how strange this situation is, consider the Einstein-Hilbert action,

$$
S=\int \sqrt{-g}\left(\frac{1}{16 \pi G} R+\frac{\Lambda}{8 \pi G}\right)
$$

Here, the first term describes the response of the total action to any deformation causing curvature. This response is huge, since Newton's constant, which is tiny, occurs in the denominator. In contrast, the second term describes the response of the total action upon scaling. This response is very tiny, since the cosmological constant $\Lambda$ is extremely small - indeed it was thought to vanish altogether until recently.

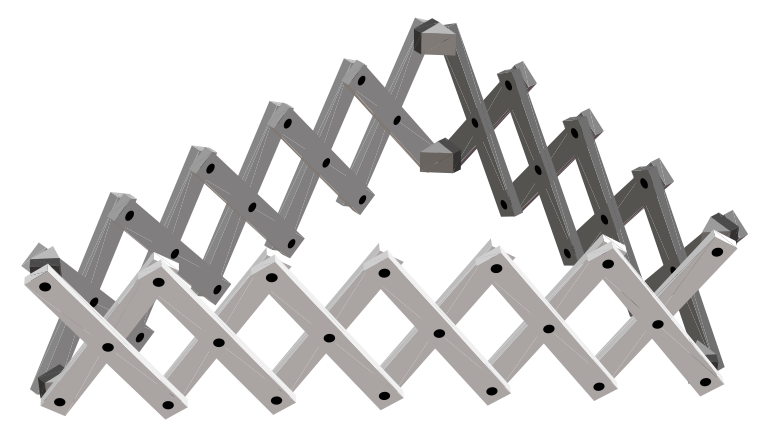

Figure 1: The "fabric of space-time", with tiny cosmological constant. Explanation: see text.

In Fig. 1, a piece of fabric is sketched with similar properties in ordinary 3-space. Globally, this material allows for stretching and squeezing with relatively little resistance, but changing the ratios of the sides of the large triangle, or its angles, requires much more force. One could build 
more elaborate structures from these basic triangular units, such that their shapes are fixed, but their sizes not. An engineer would observe, however, that even if the hinges and the rods were made extremely strong and sturdy, resistance against changes of shape would still be rather weak. In the limit where the sizes of the structures are very large compared to those of the hinges, resistance against changes of shape would dwindle.

Comparing this with the situation in our universe shows in a more tangible way how odd it is that, a term with dimensions as low as the cosmological constant, can nevertheless be so tiny (120 factors of 10) compared with the much higher dimensional Einstein-Hilbert term. This oddity is the main reason why all attempts to find a natural explanation of this feature have failed. Unless one is prepared to accept the anthropic argument ("the universe is like this because all other universes are uninhabitable for intelligent beings"), a more drastic approach will be needed. Here again, we emphasize that, in any more advanced theory for Planck length physics, the definition of what exactly the vacuum state is, will have to require special attention. It could be that one has to define that the vacuum state is the one in which 3-space is as flat as it can be. One then again is confronted with the problem to understand why all other physical states have not only positive energy, but also energy densities that are bounded from below.

Note that, in conventional quantum mechanics, the Hamiltonian plays a dual role: on the one hand it is simply the operator that generates the equations for evolution in time, while on the other hand it stabilizes the ground state, or vacuum. Energy conservation prevents small fluctuations to grow, because there are no other states where the total energy vanishes. One-particle states are also stable because there are no other states with matching energy and momentum, and this situation is guaranteed only because all energies are bounded from below. This is why the lower bound on energy is an absolutely vital feature of conventional quantum mechanics. It must be reproduced, whenever an 'underlying' theory is proposed.

\section{Gauge- and diffeomorphism invariance as emergent symmetries}

Most likely, however, the hideously tiny value of the cosmological constant is pointing towards a deeper kind of misunderstanding concerning diffeomorphism invariance in gravity. A remote possibility is suggested by our theory where quantum mechanical effects are generated as an emergent 
phenomenon in a world that is deterministic at the Planck scale. Information loss leads to a description of physical states forming equivalence classes. As stated, the equivalence classes are very large; when black holes are formed, the equivalence classes assemble on the surface area of the horizon, while the original ontological states are defined in the bulk of 3space. If information loss forces two states to evolve identically, the states are said to sit in one equivalence class.

Even if one would not buy the idea that there is an underlying deterministic theory, one could suspect that these equivalence classes can be described as gauge-equivalence classes. The transition from one element to another element of an equivalence classes is a local gauge transformation. If so, then local gauge invariance will not be a property of the underlying theory, but an emergent phenomenon.

This naturally begs us to question: could diffeomorphism invariance be also just such a symmetry? Could it be that two states that differ from one another just by a local coordinate transformation, sit in one equivalence class, which would mean that they could evolve into the same final state? This might be possible. It would mean that the original, deterministic theory might require a preferred coordinate frame, which however would wash away due to information loss. The preferred coordinate frame might naturally select a flat space-time as a ground state solution, and thus a curvature-free configuration would be selected as the natural vacuum state.

Needless to say, this argument is hopelessly inadequate to solve the cosmological constant problem, but it could serve to shed a different light on it. It illustrates that there may be more, unconventional directions to search for a solution to the problem of reconciling quantum mechanics with general relativity.

\section{References}

[1] B.S. DeWitt, Phys. Rev. Lett. 12 (1964) 742, and Phys. Rev. 160 (1967) 1113; ibid. 162 (1967) 1195, 1239;

M.J. Duff, Covariant Quantization, in Quantum Gravity, and Oxford Symposium, C.J. Isham et al, eds., Clarendon Press, Oxford, 1975, p. 78; S. Deser, Quantum Gravitation: Trees, Loops and Renormalization, ibid., p. 136;

G. 't Hooft, Perturbative Quantum Gravity, in Proceedings of the International School of Subnuclear Physics, Erice 2002, From Quarks 
and Gluons to Quantum Gravity, Subnuclear Series Vol. 40, ed. A. Zichichi, World Scientific, p. 249.

[2] R. Penrose, The Emperor's New Mind: Concerning Computers, Minds, and the Laws of Physics, Oxford University Press, 1989.

[3] G. 't Hooft, A Confrontation with Infinity, Nobel Lecture in Physics 1999, Rev. Mod. Phys. 72 (2000) 333. In slightly modified form: in Proceedings of the International Conference on Fundamental and Applied Aspects of Modern Physics, S.H. Connel and R. Tegen, eds, World Scientific, 2001, p. 317.

[4] M.B. Green, J.H. Schwarz and E. Witten, Superstring Theory, Vols. I and II, Cambridge Univ. Press, 1987;

J. Polchinski, String Theory, Vols. I and II, Cambridge Univ. Press, 1998.

[5] A. Ashtekar et al, Class. Quantum Grav. 6 (1989) L185; id., Phys. Rev. Lett. 69 (1992) 237;

C. Rovelli and L. Smolin, Phys. Rev. Lett. 61 (1988) 1155, Nucl Phys. B133 (1990) 80; C. Rovelli, Class. Quant. Grav. 8 (1991) 297; 317 ;

T. Thiemann, textitClass. Quant. Grav. 15 (1998) 1207.

[6] A. Staruszkiewicz, Acta Phys. Polon. 24 (1963) 734; S. Deser, R. Jackiw and G. 't Hooft, Ann. Phys. 152 (1984) 220; G. 't Hooft, Class. Quantum Grav. 10 (1993) 1023.

[7] E. Witten, Nucl. Phys. B311 (1988) 46; S. Carlip, Nucl. Phys. B324 (1989) 106, and in: Physics, Geometry and Topology, NATO ASI series B, Physics, Vol. 238, H.C. Lee ed., Plenum 1990, p. 541.

[8] G. 't Hooft, The Holographic Mapping of the Standard Model onto the Black Hole Horizon, Part I: Abelian Vector Field, Scalar Field and BEH Mechanism, Class. Quant. Grav. 22 (2005) 4179, ITP-UU05/17, SPIN-05/13, gr-qc/0504120 (Note: some sign corrections and further elucidations in the published version).

[9] J. Maldacena, Adv. Theor. Math. Phys. 2 (1998) 231; hepth/9711200; Phys. Rev. Lett. 80 (1998) 4859; hep-th/9803002.

[10] G. 't Hooft, Can Quantum mechanics be reconciled with Cellular Automata?, in Digital Perspectives, Int. J. Theor. Physics 42 (2003) 349 . 
[11] G. 't Hooft, Quantum Gravity as a Dissipative Deterministic System, Class. Quant. Grav. 16 (1999) 3263 (gr-qc/9903084); id., Quantum Mechanics and determinism, in Proceedings of the Eighth Int. Conf. on Particles, Strings and Cosmology, Univ. of North Carolina, Chapel Hill, Apr. 10-15, 2001, P. Frampton and J. Ng, Eds., Rinton Press, Princeton, p. 275; hep-th/0105105.

[12] S. Nobbenhuis, Categorizing different approaches to the cosmological constant problem, ITP-UU-04-40, SPIN-04-23, Nov 2004, grqc/0411093, Found. Mod. Phys., to be pub. 